\section{Independent Variable}

Sven Hilbert

Department of Psychology, Psychological

Methods and Assessment, München, Germany

Faculty of Psychology, Educational Science, and

Sport Science, University of Regensburg,

Regensburg, Germany

\section{Synonyms}

Covariable

\section{Definition}

The independent variable is a variable whose variation is statistically related to variation in the dependent variable.

\section{Short Summary}

In the investigation of covariation between variables, they are usually labeled as either "independent" or "dependent" (variables). In this context, variation in the independent variable(s) is produced optimally in a systematic manner - while the effect of this variation on the dependent variable(s) is observed. Since the direction of causation is not necessarily clear (i.e., which variable causes variation and which one depends on variation in the other) and a direct causal relationship between the variables need not be present at all, the labeling of the variables has to be defined by theoretical assumptions postulated by the researcher investigating the relationship.

The independent variable is particularly important in experimental settings: experiments are defined by the systematic variation of an independent variable, which creates different experimental conditions defined by the different variations (states) of the independent variable. Differences in the dependent variable are then compared between the experimental conditions to draw conclusions about the relationship in question.

For a more extensive description of independent variables, see $>$ Dependent Variable.

\section{Cross-References}

Dependent Variable 